Eric Van Cutsem and Sabine Tejpar, University Hospitals Leuven and Katholieke Universiteit Leuven, Leuven, Belgium; Heinz-Josef Lenz, University of Southern California Norris Comprehensive Cancer Center, Los Angeles, CA; Claus-Henning Köhne, Klinikum Oldenburg, Oldenburg; Volker Heinemann, University Hospital Grosshadern Munich; Ivan Melezínek, Frank Beier, and Christopher Stroh, Merck KGaA, Darmstadt, Germany; Philippe Rougier, Paris Descartes University, Hôpital Européen Georges Pompidou, Paris, France; J. Han van Krieken, Radboud University Nijmegen Medical Center, Nijmegen, the Netherlands; and Fortunato Ciardiello, Second University of Naples, Naples, Italy.

Published online ahead of print at www.jco.org on January 20, 2015

Processed as a Rapid Communication manuscript.

Supported by Merck KGaA, which also funded medical writing services.

Presented in part at the 50th Annual Meeting of the American Society of Clinical Oncology, Chicago, IL, May 30-June 3, 2014, and European Society for Medical Oncology 16th World Congress on Gastrointestinal Cancer, Barcelona, Spain, June 25-28, 2014.

Authors' disclosures of potential conflicts of interest are found in the article online at www.jco.org. Author contributions are found at the end of this article.

Clinical trial information: NCT00154102.

Corresponding author: Eric Van Cutsem, MD, PhD, Digestive Oncology, University Hospitals Gasthuisberg/Leuven, Herestraat 49, 3000 Leuven, Belgium; e-mail: eric.vancutsem@ uzleuven.be.

(c) 2015 by American Society of Clinical Oncology

0732-183X/15/3307w-692w/\$20.00 DOI: $10.1200 / J C O .2014 .59 .4812$

\title{
Fluorouracil, Leucovorin, and Irinotecan Plus Cetuximab Treatment and RAS Mutations in Colorectal Cancer
}

Eric Van Cutsem, Heinz-Josef Lenz, Claus-Henning Köhne, Volker Heinemann, Sabine Tejpar, Ivan Melezinek, Frank Beier, Christopher Stroh, Philippe Rougier, J. Han van Krieken, and Fortunato Ciardiello

$$
\begin{array}{llllllll}
\text { A } & \text { B } & \text { S } & \text { T } & \text { R } & \text { A } & \text { C } & \text { T }
\end{array}
$$

\section{Purpose}

The phase III CRYSTAL study demonstrated that addition of cetuximab to fluorouracil, leucovorin, and irinotecan (FOLFIRI) significantly improved overall survival, progression-free survival, and objective response in the first-line treatment of patients with KRAS codon 12/13 (exon 2) wild-type metastatic colorectal cancer ( $\mathrm{mCRC}$ ). Outcome was reassessed in subgroups defined by extended RAS mutation testing.

\section{Patients and Methods}

Existing DNA samples from KRAS exon 2 wild-type tumors from CRYSTAL study patients were reanalyzed for other RAS mutations in four additional KRAS codons (exons 3 and 4) and six NRAS codons (exons 2, 3, and 4) using beads, emulsion, amplification, and magnetics technology. No tissue microdissection was performed. $A \geq 5 \%$ mutant allele cutoff was used to call mutations.

\section{Results}

Mutation status was evaluable in 430 (64.6\%) of 666 patients with KRAS exon 2 wild-type tumors. Other RAS mutations were detected in $63(14.7 \%)$ of 430 patients. In those with RAS wild-type tumors, a significant benefit across all efficacy end points was associated with the addition of cetuximab to FOLFIRI. In patients with other RAS tumor mutations, no difference in efficacy outcomes between treatment groups was seen. The safety profile in $R A S$ subgroups was similar and in line with expectations.

\section{Conclusion}

In the first-line treatment of $\mathrm{mCRC}$, patients with RAS wild-type tumors derived a significant benefit from the addition of cetuximab to FOLFIRI; patients with RAS tumor mutations did not. Molecular testing of tumors for all activating RAS mutations is essential before considering anti-epidermal growth factor receptor therapy, thereby allowing the further tailoring of cetuximab administration to maximize patient benefit.

\section{J Clin Oncol 33:692-700. (C) 2015 by American Society of Clinical Oncology}

\section{INTRODUCTION}

The randomized phase III CRYSTAL (Cetuximab Combined With Irinotecan in First-Line Therapy for Metastatic Colorectal Cancer) study showed that the addition of cetuximab to infusional fluorouracil, leucovorin, and irinotecan (FOLFIRI) significantly improved overall survival (OS) time, progression-free survival (PFS) time, and objective response rate in the first-line treatment of patients with KRAS codon 12 and 13 (hereinafter exon 2) wild-type metastatic colorectal cancer (mCRC). No cetuximab efficacy benefit was apparent in the subgroup of patients whose tumors carried such exon 2 mutations. ${ }^{1,2}$

In the same setting, the randomized phase II OPUS (Oxaliplatin and Cetuximab in First-Line Treatment of Metastatic Colorectal Cancer) study demonstrated that the addition of cetuximab to another standard first-line regimen-fluorouracil, leucovorin, and oxaliplatin (FOLFOX4) — significantly improved objective response rate and PFS time in patients with KRAS exon 2 wild-type tumors. However, it was reported that for patients with KRAS exon 2 tumor mutations, the addition of cetuximab to FOLFOX4 resulted in worse outcome compared with FOLFOX4 alone. ${ }^{3,4}$ Similar findings were also reported from the PRIME (Panitumumab Randomized Trial in Combination With Chemotherapy for Metastatic Colorectal Cancer to Determine Efficacy) study for another epidermal growth factor receptor (EGFR) antibody, panitumumab, which improved outcome in patients when combined with FOLFOX4 in the first-line treatment of KRAS exon 2 wild-type mCRC. As for cetuximab, a negative effect 
was apparent when panitumumab was combined with FOLFOX4 in patients with KRAS exon 2 mutations. ${ }^{5}$

Activating missense mutations of KRAS at codons other than 12 or 13 have been identified in a wide range of tumor types, including CRC. ${ }^{6}$ Similar somatic tumor mutations have also been detected at corresponding loci within the NRAS gene. A retrospective analysis of the PRIME study demonstrated that a subgroup of $17.4 \%$ of patients with KRAS exon 2 wild-type mCRC had tumor mutations at another RAS locus (KRAS codons 61, 117, and 146; NRAS codons 12, 13, and $61)$. Such other RAS mutations were associated negatively with outcome in patients receiving FOLFOX4 plus panitumumab. ${ }^{7}$ Exploratory analysis further suggested that RAS codon 59 mutations might also be negative biomarkers for panitumumab efficacy.

The primary objective of our post hoc investigation was to assess the treatment effect of FOLFIRI plus cetuximab compared with FOLFIRI alone in patients with tumors carrying predefined mutations at RAS loci other than KRAS codon 12 or 13 (other RAS mutations). Also assessed was the treatment effect in patients with evaluable tumors wild type at all RAS loci.

\section{PATIENTS AND METHODS}

\section{Study Design and Treatment}

The phase III CRYSTAL study compared 14-day cycles of FOLFIRI plus weekly cetuximab with FOLFIRI alone as first-line treatment for patients with EGFR-expressing mCRC. Treatment was continued until disease progression, unacceptable toxicity, or withdrawal of consent. The primary end point was PFS, as determined by independent review. The clinical study was approved by the independent ethics committee of each trial center and was carried out in accordance with the Declaration of Helsinki. Eligibility criteria have been described previously. ${ }^{1}$ All patients provided informed consent before inclusion. A previous retrospective subgroup analysis investigated the association of tumor KRAS exon 2 mutation status and treatment outcome. ${ }^{2}$

\section{RAS Mutation Testing}

Extended RAS mutation testing was performed on DNA samples extracted previously from formalin-fixed paraffin-embedded tumor tissue sections from CRYSTAL study patients and which had been scored in earlier investigations as wild type at codons 12 and 13 of KRAS. ${ }^{2}$ Before DNA extraction, stained slides were reviewed by a pathologist to estimate overall neoplastic cell content; no exclusion criteria were applied. The estimated fraction of neoplastic cells was between $5 \%$ and $60 \%$ for most samples. Because the polymerase chain reaction clamping and melting curve technique used in the original testing was a highly sensitive method designed to enrich for mutant over wild-type sequences, ${ }^{1,8}$ macro- or microdissection of tissue sections was not carried out. In the application of the $5 \%$ cutoff in our analysis, the estimated fraction of neoplastic cells was not taken into consideration. Ploidy status, which was not assessed, was also not taken into consideration.

The highly sensitive beads, emulsion, amplification, and magnetics (BEAM) technology was selected for the RAS mutation analysis to take into account the source and nature of the tumor DNA available (Data Supplement). ${ }^{9}$ This approach can detect and enumerate mutant versus wild-type DNA sequences at ratios down to $1: 10,000(0.01 \%) .{ }^{10,11}$ RAS testing was carried out by a contract research organization (Sysmex Inostics, Hamburg, Germany).

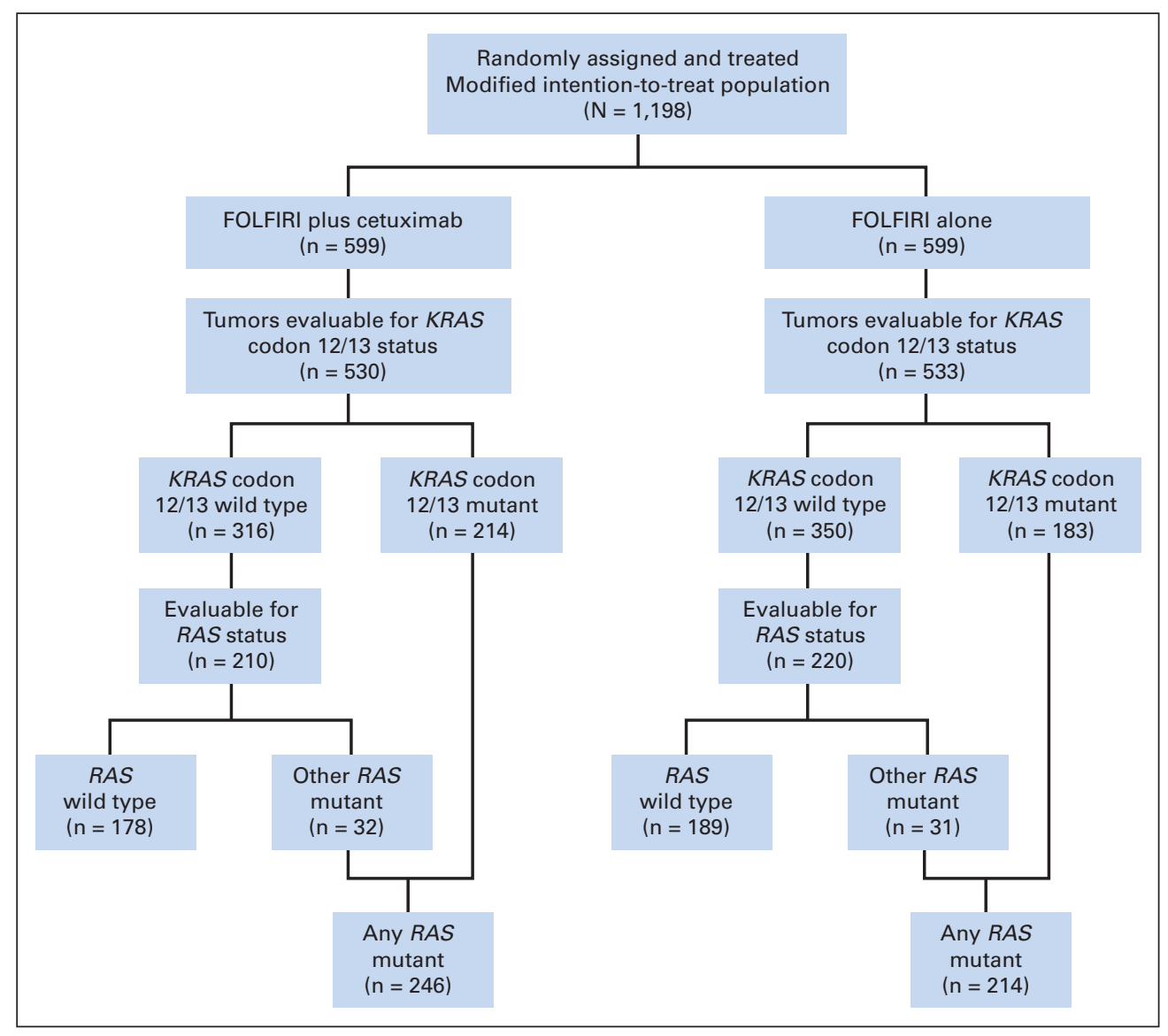

Fig 1. Study profile. FOLFIRI, fluorouracil, leucovorin, and irinotecan. 
Table 1. Baseline Demographic and Clinical Characteristics of KRAS Exon 2 Wild-Type Subgroup of KRAS Population and RAS Subpopulations

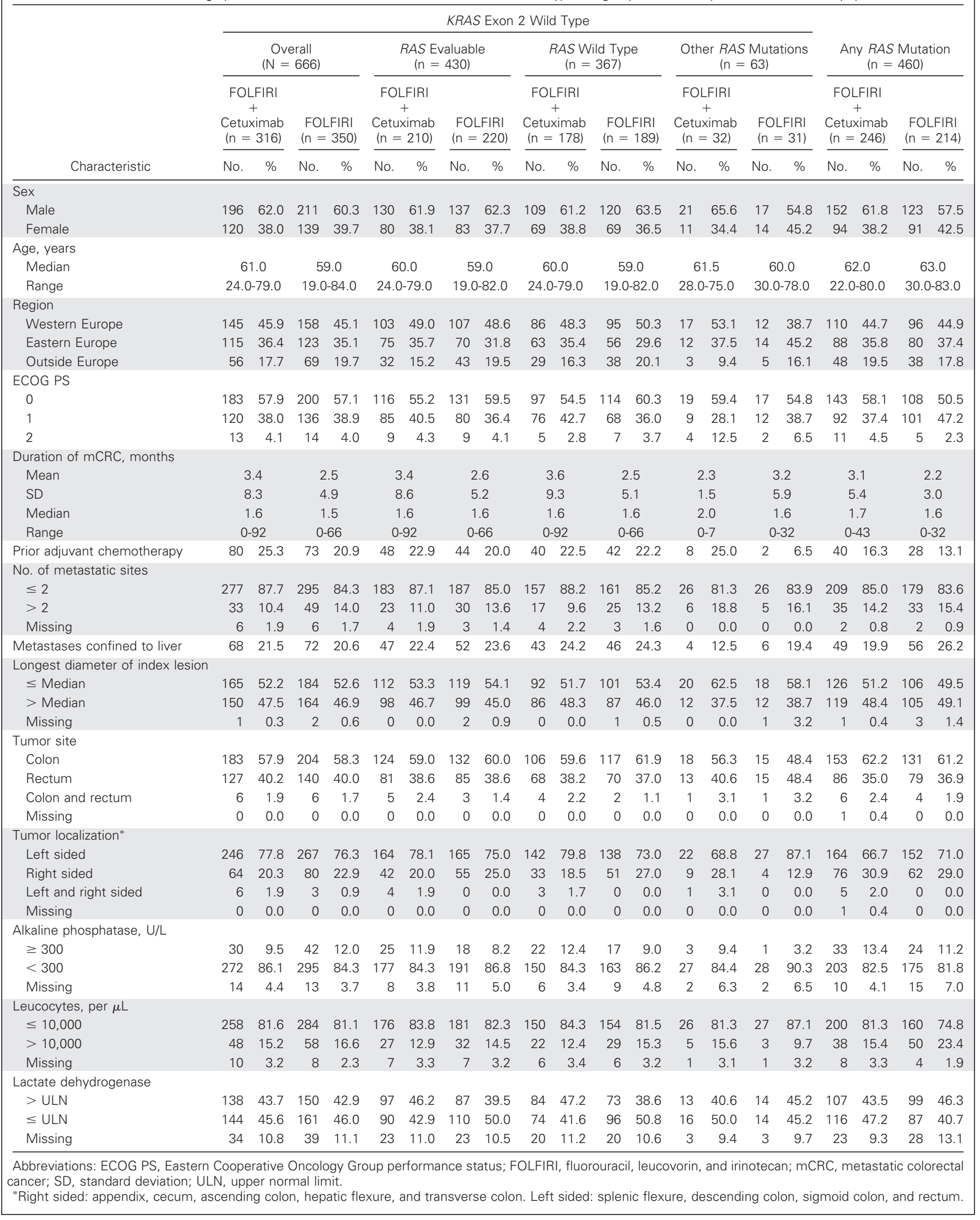




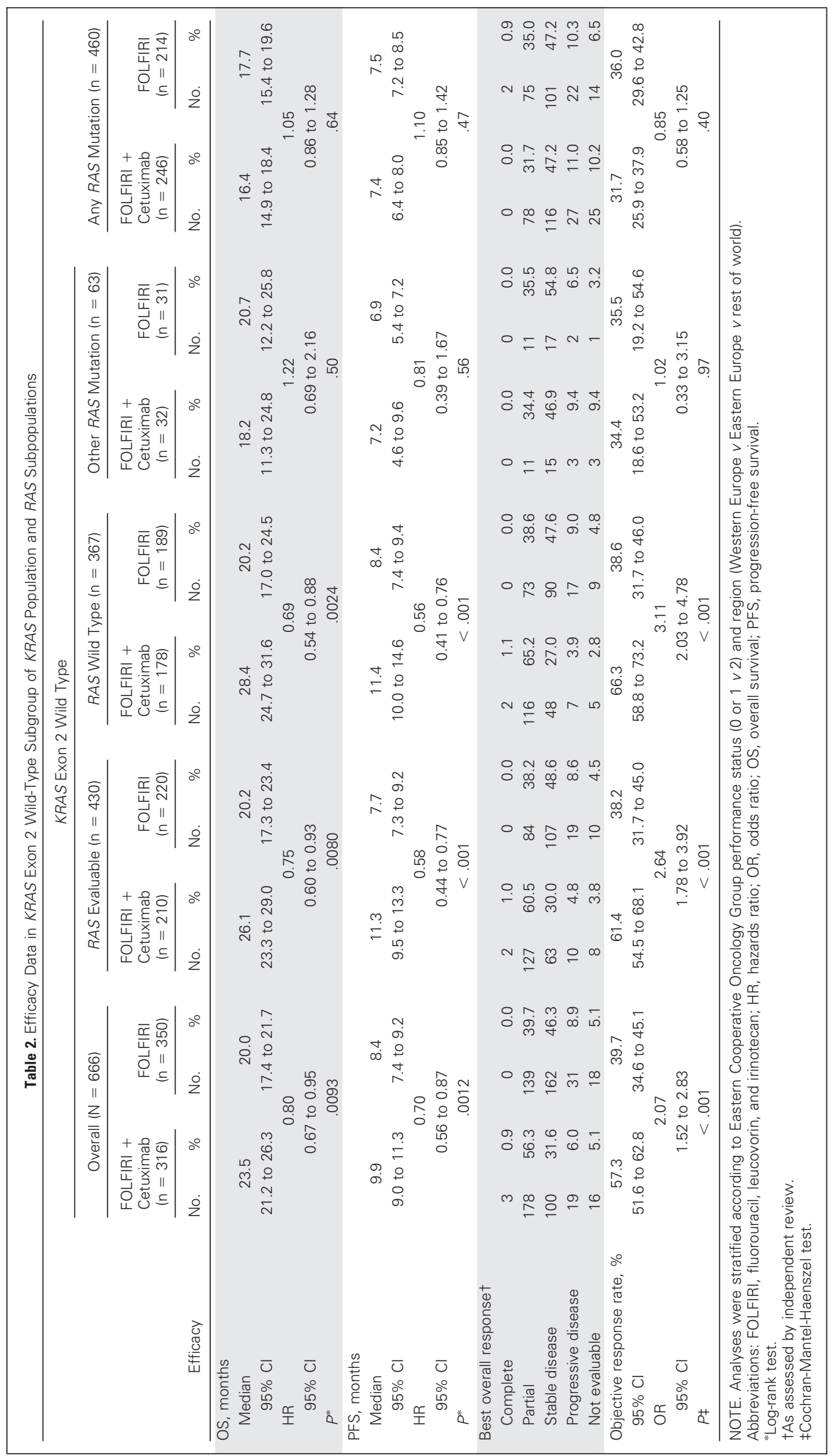


The presence of 26 specific mutations reported in the Catalogue of Somatic Mutations in Cancer and/or Cancer Genome Atlas databases within KRAS exons 3 (codons 59 and 61) and 4 (codons 117 and 146) and NRAS exons 2 (codons 12 and 13), 3 (codons 59 and 61), and 4 (codons 117 and 146) was investigated (Data Supplement). Samples were not reassessed for the presence of KRAS exon 2 (codons 12 and 13) mutations.

\section{Statistical Analysis}

For each evaluable sample, the fraction of mutant RAS alleles was calculated by dividing the number of mutant beads by the total number of beads with polymerase chain reaction product (sum of mutant, wild-type, and mutant/wild-type beads). Broadly in line with the sensitivity of other approaches that might be used clinically to assign RAS mutation status, and after an initial evaluation of the impact of using alternative cutoff values, tumors were scored as RAS mutant if the sum of the individual percentages of mutant sequences over total amplified sequences for the analyzed loci was $\geq 5 \%$, regardless of whether all loci were evaluable for mutation status. Tumors were scored as $R A S$ wild type only if all 26 mutation assays were evaluable and the summed prevalence of mutations across all loci was $<5 \%$.

The primary objective of our post hoc analysis was to investigate the treatment effect of FOLFIRI plus cetuximab compared with FOLFIRI alone in patients with tumor RAS mutations other than KRAS exon 2 (other RAS). This extended analysis was limited to those CRYSTAL study patients for whom an evaluable tumor DNA sample was available. Outcome for patients with tumors wild type at all tested RAS loci was also investigated. Treatment outcome in patients with any RAS mutation, comprising those with either previously identified KRAS exon 2 mutations (not reassessed in our study) or other RAS mutations, was also analyzed. Outcome in patients wild type for both $R A S$ and $B R A F\left(V 600 \mathrm{E}\right.$; as previously defined ${ }^{2}$ ) was considered. The treatment effect in different other RAS-mutant populations, as defined according to a range of diagnostic cutoffs from $0.1 \%$ to $20 \%$, was visualized using forest plots of hazard ratios (HRs) and odds ratios (ORs).

HRs and ORs were calculated for FOLFIRI plus cetuximab versus FOLFIRI alone. For OS and PFS, HRs and 95\% CIs for treatment comparisons were calculated using univariable Cox proportional hazards models. Median survival times were estimated using the Kaplan-Meier method ${ }^{12}$ (productlimit estimates), and $P$ values were calculated using log-rank tests. For objective response, treatment groups were compared using Cochran-MantelHaenszel tests. All analyses, apart from those for different cutoff values as presented in the forest plots, were stratified according to Eastern Cooperative Oncology Group performance status and region, as assigned through the interactive voice response system at random assignment.

\section{RESULTS}

\section{Patients and Samples}

Of the 666 CRYSTAL study patients with tumors previously typed as KRAS exon 2 wild type, RAS tumor mutation status was evaluable in 430 (64.6\%; Fig 1). Samples from the remaining 236 patients were not evaluable, either because of insufficient residual DNA or because of the failure of $\geq 1$ mutation assay. Using a $5 \%$ mutant/wild-type cutoff, other RAS mutations were scored in 63 (14.7\%) of 430 patients. Those with other RAS mutations were grouped with patients previously classified as having tumors with KRAS exon 2 mutations $(\mathrm{n}=397)$ to comprise a combined population of patients with any $R A S$ mutations $(\mathrm{n}=460)$. Using the less stringent cutoff of $\geq 0.1 \%$ mutant/wild-type sequences, other $R A S$ mutations were scored in $86(20.0 \%)$ of 430 patients. The most common site of other RAS mutations was within KRAS exon 4 (Data Supplement).

Of 422 RAS/BRAF evaluable tumors, 46 (10.9\%) were known to carry BRAF mutations, all but one of which were scored as RAS wild type; 315 (74.6\%) of 422 tumors were wild type for both $R A S$ and $B R A F$.

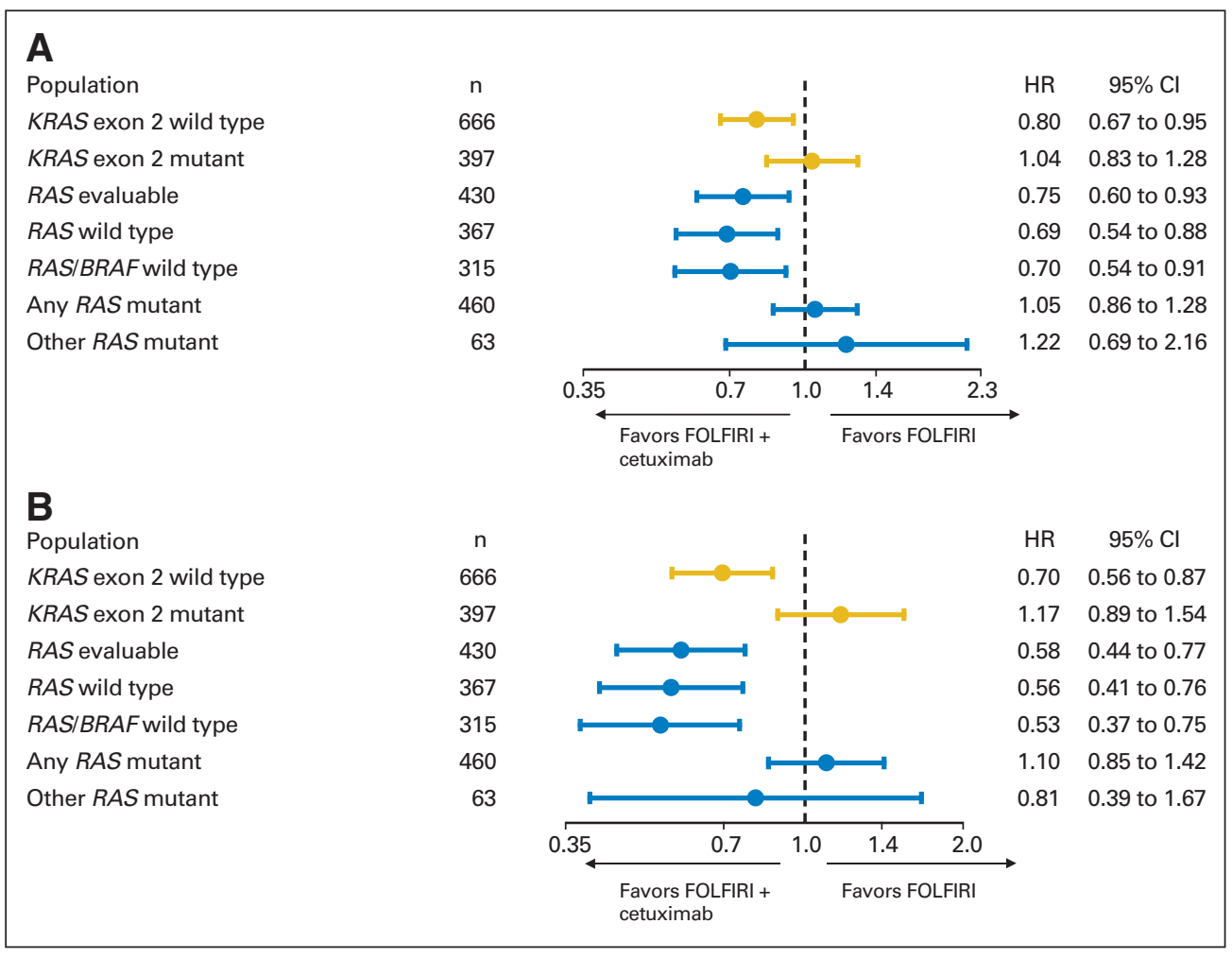

Fig 2. Hazard ratios (HRs) for (A) overall and (B) progression-free survival according to tumor KRAS exon 2 and RAS mutation status. FOLFIRI, fluorouracil, leucovorin, and irinotecan. 


\section{Comparability of Evaluable Populations}

Baseline characteristics of the RAS-evaluable population and $R A S$ wild-type and other $R A S$-mutant subgroups were broadly similar to those of the KRAS exon 2 wild-type subgroup of the KRAS population (Table 1). However, and although balanced between treatment groups, notably fewer patients with a tumor RAS mutation (68 [14.8\%] of 460) had received prior adjuvant chemotherapy compared with those with RAS wild-type tumors (82 [22.3\%] of 367).

In relation to efficacy, there was a trend for better outcome for the FOLFIRI-plus-cetuximab group compared with the FOLFIRI-alone group in the RAS-evaluable population compared with the KRAS exon 2 wild-type population, with the difference most pronounced in relation to PFS and objective response (Table 2; Fig 2). Given the similarity in baseline characteristics between the KRAS exon 2 wildtype population and the RAS-evaluable subset, there was no obvious selection bias that might have explained the increased cetuximab benefit in the RAS-evaluable population.

\section{Efficacy According to RAS Mutation Status}

A clear and significant benefit associated with the addition of cetuximab to FOLFIRI was apparent in relation to OS, PFS, and objective response in patients with $R A S$ wild-type tumors ( $\mathrm{n}=$ 367; efficacy outcome summarized in Table 2 and Figs 2 to 4 ). The HR for OS time and OR for objective response rate were more favorable toward FOLFIRI plus cetuximab in the RAS wild-type population than in the $R A S$-evaluable population. Setting aside the possible issue of selection bias, results for the RAS wild-type/BRAF wild-type population were similar to those of the $R A S$ wild-type population (Data Supplement). For patients with tumors harboring other RAS mutations $(\mathrm{n}=63)$, no clear cetuximab treatment benefit was apparent. Efficacy outcomes were also investigated for the group of patients with any RAS mutation $(\mathrm{n}=460)$. No clear difference in efficacy outcome between treatment groups was apparent in this population.

BEAMing analysis allowed for the detection of tumor RAS mutations at low prevalence. Using a more sensitive threshold of $0.1 \%$ to call mutations, $23(5.3 \%)$ of 430 tumors classified as RAS wild type when using a 5\% cutoff were instead scored as RAS mutant. Efficacy outcomes were reassessed in RAS wild-type and $R A S$-mutant populations, as defined according to this $0.1 \%$ cutoff (Data Supplement). As for the 5\% cutoff analysis, HRs and ORs did

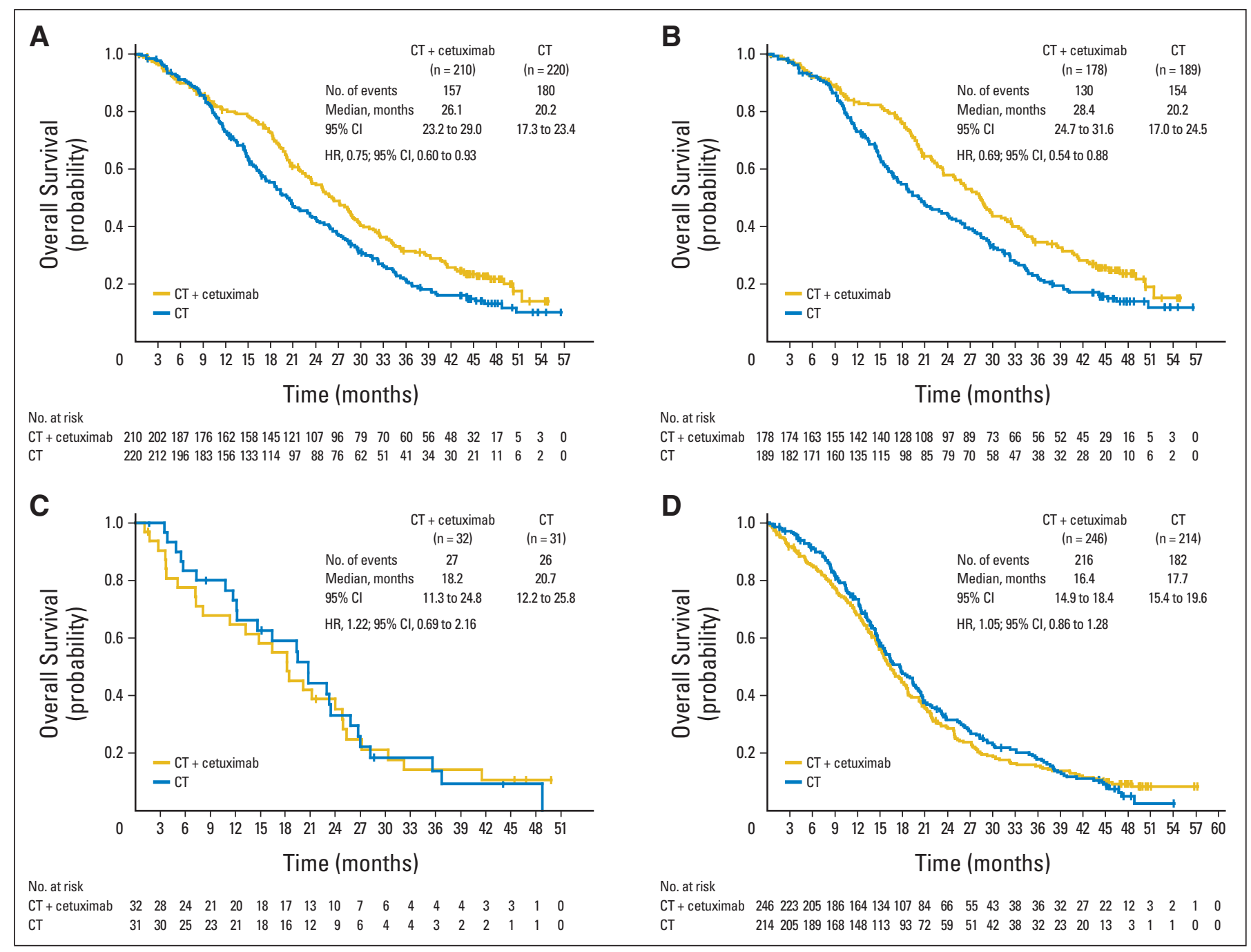

Fig 3. Overall survival according to treatment group in RAS populations. (A) KRAS codon 12 or 13 wild type, evaluable for other RAS mutations. (B) RAS wild type (all loci). (C) KRAS codon 12 or 13 wild type; other RAS mutations. (D) RAS mutation (any locus). CT, chemotherapy; HR, hazard ratio. 

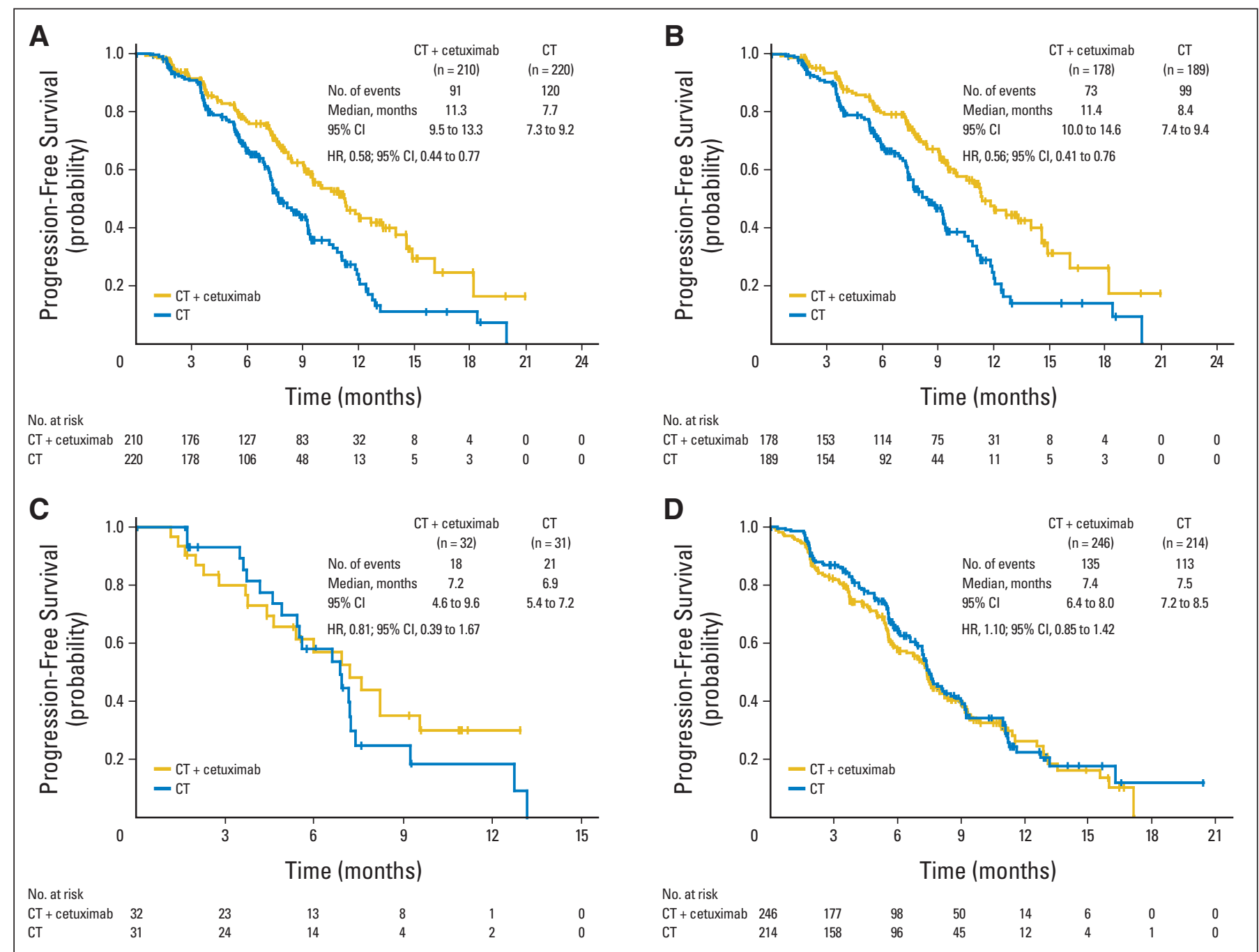

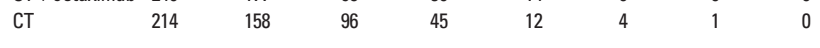

Fig 4. Progression-free survival according to treatment group in RAS populations. (A) KRAS codon 12 or 13 wild type, evaluable for other RAS mutations. (B) RAS wild type (all loci). (C) KRAS codon 12 or 13 wild type; other RAS mutations. (D) RAS mutation (any locus). CT, chemotherapy; HR, hazard ratio.

not suggest a positive or negative effect on efficacy associated with the addition of cetuximab to FOLFIRI in patients with tumor RAS mutations.

Treatment outcome in other RAS-mutant populations defined according to cutoff values of $20 \%, 10 \%, 5 \%, 1 \%$, and $0.1 \%$ were visualized using forest plots of HRs for OS and PFS times and ORs for objective response rates (Data Supplement). These plots suggested that there was a relationship between the fraction of $R A S$-mutated neoplastic cells in a tumor and the strength of EGFR-targeted therapy resistance and that patients with other tumor RAS mutation signals between $0.1 \%$ and $<5 \%$ may have benefited from the addition of cetuximab to FOLFIRI.

\section{Safety}

The overall incidence of adverse events according to treatment group was broadly similar across the KRAS and RAS subgroups (Table 3 ). In addition, the incidence of commonly reported adverse events in each treatment group was also generally similar across these populations and in line with expectations.

\section{DISCUSSION}

The aim of our post hoc analysis of the CRYSTAL study was to investigate the impact of RAS mutations other than KRAS codon 12 or 13 in relation to treatment effects. In the evaluable patients with other tumor RAS mutations, there was no clear evidence that the addition of cetuximab to FOLFIRI modified efficacy outcome. However, given the relatively small number of patients in this group, no definitive conclusions on the negative predictive value of other RAS mutations can be drawn. In the combined group of patients with any $R A S$ mutation (KRAS exon 2 or other RAS), there was no indication that the addition of cetuximab to FOLFIRI either improved or worsened outcome. The absence of a negative effect in the RAS-mutant subgroup when cetuximab was combined with FOLFIRI in our study contrasts strongly with the data from the OPUS ${ }^{13}$ and PRIME $^{7}$ RAS investigations, which suggested worse outcome in the RAS-mutant subgroups associated with the addition of cetuximab (PFS: HR, 1.54; 95\% CI, 1.04 to 2.29 ) or panitumumab (PFS: HR, 1.31; 95\% CI, 1.07 


\begin{tabular}{|c|c|c|c|c|c|c|c|c|c|c|c|c|c|c|c|c|c|c|c|c|}
\hline \multirow[b]{4}{*}{$A E$} & \multicolumn{16}{|c|}{ KRAS Exon 2 Wild Type } & \multirow{2}{*}{\multicolumn{4}{|c|}{$\begin{array}{l}\text { Any RAS Mutation } \\
\quad(n=460) \dagger\end{array}$}} \\
\hline & \multicolumn{4}{|c|}{$\begin{array}{c}\text { Overall } \\
(\mathrm{N}=666)^{*}\end{array}$} & \multicolumn{4}{|c|}{$\begin{array}{l}\text { RAS Evaluable } \\
(n=430) \dagger\end{array}$} & \multicolumn{4}{|c|}{$\begin{array}{l}\text { RAS Wild Type } \\
(\mathrm{n}=367) \dagger\end{array}$} & \multicolumn{4}{|c|}{$\begin{array}{l}\text { Other RAS Mutation } \\
\qquad(\mathrm{n}=63) \dagger\end{array}$} & & & & \\
\hline & \multicolumn{2}{|c|}{$\begin{array}{c}\text { FOLFIRI } \\
+ \\
\text { Cetuximab } \\
(\mathrm{n}=316) \\
\end{array}$} & \multicolumn{2}{|c|}{$\begin{array}{l}\text { FOLFIRI } \\
(\mathrm{n}=350) \\
\end{array}$} & \multicolumn{2}{|c|}{$\begin{array}{c}\text { FOLFIRI } \\
+ \\
\text { Cetuximab } \\
(n=210)\end{array}$} & \multicolumn{2}{|c|}{$\begin{array}{l}\text { FOLFIRI } \\
(n=220) \\
\end{array}$} & \multicolumn{2}{|c|}{$\begin{array}{c}\text { FOLFIRI } \\
+ \\
\text { Cetuximab } \\
(n=178) \\
\end{array}$} & \multicolumn{2}{|c|}{$\begin{array}{l}\text { FOLFIRI } \\
(\mathrm{n}=189) \\
\end{array}$} & \multicolumn{2}{|c|}{$\begin{array}{c}\text { FOLFIRI } \\
+ \\
\text { Cetuximab } \\
(\mathrm{n}=32) \\
\end{array}$} & \multicolumn{2}{|c|}{$\begin{array}{l}\text { FOLFIRI } \\
(n=31) \\
\end{array}$} & \multicolumn{2}{|c|}{$\begin{array}{c}\text { FOLFIRI } \\
+ \\
\text { Cetuximab } \\
(\mathrm{n}=246) \\
\end{array}$} & \multicolumn{2}{|c|}{$\begin{array}{l}\text { FOLFIRI } \\
(\mathrm{n}=214) \\
\end{array}$} \\
\hline & No. & $\%$ & No. & $\%$ & No. & $\%$ & No. & $\%$ & No. & $\%$ & No. & $\%$ & No. & $\%$ & No. & $\%$ & No. & $\%$ & No. & $\%$ \\
\hline Any & 257 & 81.1 & 211 & 60.3 & 169 & 80.5 & 131 & 59.5 & 144 & 80.9 & 110 & 58.2 & 25 & 78.1 & 21 & 67.7 & 189 & 76.8 & 133 & 62.1 \\
\hline \multicolumn{21}{|l|}{ MedDRA preferred term } \\
\hline Neutropenia & 97 & 30.6 & 83 & 23.7 & 64 & 30.5 & 46 & 20.9 & 55 & 30.9 & 38 & 20.1 & 9 & 28.1 & 8 & 25.8 & 62 & 25.2 & 58 & 27.1 \\
\hline Diarrhea & 52 & 16.4 & 35 & 10.0 & 29 & 13.8 & 20 & 9.1 & 26 & 14.6 & 18 & 9.5 & 3 & 9.4 & 2 & 6.5 & 30 & 12.2 & 22 & 10.3 \\
\hline Rash & 28 & 8.8 & 0 & 0.0 & 19 & 9.0 & 0 & 0.0 & 16 & 9.0 & 0 & 0.0 & 3 & 9.4 & 0 & 0.0 & 19 & 7.7 & 0 & 0.0 \\
\hline Leucopenia & 25 & 7.9 & 17 & 4.9 & 15 & 7.1 & 10 & 4.5 & 15 & 8.4 & 7 & 3.7 & 0 & 0.0 & 3 & 9.7 & 12 & 4.9 & 13 & 6.1 \\
\hline Fatigue & 14 & 4.4 & 20 & 5.7 & 12 & 5.7 & 11 & 5.0 & 12 & 6.7 & 9 & 4.8 & 0 & 0.0 & 2 & 6.5 & 16 & 6.5 & 5 & 2.3 \\
\hline Deep vein thrombosis & 16 & 5.0 & 2 & 0.6 & 11 & 5.2 & 2 & 0.9 & 11 & 6.2 & 1 & 0.5 & 0 & 0.0 & 1 & 3.2 & 2 & 0.8 & 9 & 4.2 \\
\hline Dermatitis acneiform & 16 & 5.0 & 0 & 0.0 & 11 & 5.2 & 0 & 0.0 & 9 & 5.1 & 0 & 0.0 & 2 & 6.3 & 0 & 0.0 & 14 & 5.7 & 0 & 0.0 \\
\hline \multicolumn{21}{|l|}{ Composite categories* } \\
\hline Any & 67 & 21.1 & 1 & 0.3 & 46 & 21.9 & 1 & 0.5 & 37 & 20.8 & 1 & 0.5 & 9 & 28.1 & 0 & 0.0 & 49 & 19.9 & 0 & 0.0 \\
\hline Acne-like rash & 52 & 16.4 & 0 & 0.0 & 36 & 17.1 & 0 & 0.0 & 30 & 16.9 & 0 & 0.0 & 6 & 18.8 & 0 & 0.0 & 41 & 16.7 & 0 & 0.0 \\
\hline $\begin{array}{l}\text { Infusion-related } \\
\text { reactions }\end{array}$ & 5 & 1.6 & 0 & 0.0 & 4 & 1.9 & 0 & 0.0 & 4 & 2.2 & 0 & 0.0 & 0 & 0.0 & 0 & 0.0 & 7 & 2.8 & 0 & 0.0 \\
\hline $\begin{array}{l}\text { NOTE. Reported at frequen } \\
\text { of special interest. } \\
\text { Abbreviations: AE, adverse } \\
\text { "According to MedDRA (ver } \\
\text { tAccording to MedDRA (ve }\end{array}$ & on 1 & $\begin{array}{l}5 \% \text { in } \\
\text { FOLFIF } \\
\text { 0.0). }\end{array}$ & eithe & uraci & te $\mathrm{s}$ & brin & $d$ & on & ild-ty & $\begin{array}{l}\text { ipe sub } \\
\text { IDRA, N }\end{array}$ & rou & safe & p & atic & & prdi & & про & $\mathrm{ca}$ & \\
\hline
\end{tabular}

to 1.60 and OS: $\mathrm{HR}, 1.25 ; 95 \% \mathrm{CI}, 1.02$ to 1.55$)$, respectively, to FOLFOX4. However, our data are in line with findings in the secondline setting, which showed that there was no negative effect of combining panitumumab with FOLFIRI for patients with KRAS exon 2-mutant or other RAS-mutant tumors. ${ }^{14,15}$ Furthermore, on the basis of the findings from these studies, current clinical guidelines recommend that only patients with $R A S$ wild-type tumors should be treated with cetuximab or panitumumab in combination with FOLFIRI or FOLFOX chemotherapy. ${ }^{16}$

In patients with $R A S$ wild-type tumors, a clear cetuximab benefit was seen across efficacy end points. However, it should be noted in this context that an increased cetuximab benefit was apparent in the RASevaluable subgroup, compared with the overall KRAS exon 2 wildtype population, regardless of $R A S$ status. Despite the similarity of baseline characteristics, selection bias in relation to the $R A S$-evaluable subgroup cannot be excluded.

The method selected to screen for RAS mutations was the BEAMing technique. ${ }^{9}$ Although this approach can detect KRAS exon 2 mutations at a level of $0.01 \%$ mutant to wild-type sequences, ${ }^{17}$ a higher threshold of $\geq 5 \%$ was used in our RAS study. This is broadly in line with the sensitivity of other techniques such as next-generation sequencing, pyrosequencing, and dideoxy nucleotide sequencing, which may be used clinically to determine RAS mutation status. Because the fraction of neoplastic cells and ploidy status were not taken into account, the percentage of mutated cells cannot be estimated.

Using this cutoff, RAS mutations were scored in $14.7 \%$ of evaluable CRYSTAL study patients with KRAS exon 2 wild-type tumors. This frequency is similar to those reported in other first-line mCRC studies, which used pyrosequencing or dideoxy sequencing/WAVE analysis. ${ }^{7,18,19}$ In common with these studies and also the parallel $R A S$ analysis of the OPUS study, ${ }^{13}$ the most common location of RAS mutations outside of KRAS exon 2 in CRYSTAL study patients was KRAS exon 4. Also of interest in relation to the mutation profile was that NRAS exon 4 tumor mutations, which were scored in four CRYSTAL study patients when using the $5 \%$ cutoff and in 12 patients when using the $0.1 \%$ cutoff, had not to our knowledge been reported previously in the tumors of patients receiving first-line treatment for mCRC. NRAS exon 4 tumor mutations were also seen in the OPUS study $R A S$ analysis, ${ }^{13}$ in which an identical BEAMing mutation detection approach was used; again, the incidence was higher when $0.1 \%$ rather than 5\% was used as a cutoff. It may be that such mutations tend to be of low prevalence and, although present, may not have been detected by other screening technologies.

Because the significance of low-prevalence KRAS or RAS mutations in relation to the effectiveness of EGFR antibody therapy in mCRC is not clear, ${ }^{20-23}$ we also explored treatment outcome in RAS subgroups defined according to a threshold of $0.1 \%$ mutant to wildtype sequences. The effect of using the lower cutoff (higher sensitivity in relation to mutation identification) was to move 23 patients previously classified as RAS wild type to the mutant group. This resulted in essentially no change in the HRs or ORs for FOLFIRI plus cetuximab over FOLFIRI alone in the revised RAS wild-type population and marginally better outcome for the FOLFIRI-plus-cetuximab group in the revised other $R A S$-mutant population. These data are therefore consistent with patients with low-prevalence mutations (between $0.1 \%$ and $<5 \%$ mutant to wild type) deriving a treatment benefit from the addition of cetuximab to FOLFIRI. This conclusion is in line with the findings from a retrospective study of 95 patients with mCRC 
who had received EGFR antibody therapy, which indicated that the PFS of patients with tumors with low-prevalence KRAS mutations $(<5 \%)$ was comparable to that of patients with KRAS wild-type tumors. ${ }^{21}$ Using higher cutoff levels resulted in too few patients for meaningful interpretations, but our forest plots suggest that the higher the fraction of mutated cells, the stronger the resistance to EGFR antibody treatment. This would be in line with the hypothesis that acquired resistance to such agents may result, at least in part, from the outgrowth of small numbers of cells with existing RAS mutations. ${ }^{20}$ The CRYSTAL study data are therefore consistent with $<5 \%$ mutant sequences being a potentially appropriate cutoff value for determining eligibility for FOLFIRI plus cetuximab as first-line treatment. Nevertheless, more data and more precise measurements of the fraction of mutated neoplastic cells, as well as an accurate definition of the association between the fraction of mutated cells and resistance to EGFR monoclonal antibodies, are needed to define the optimal cutoff for the clinical setting. This would require large collaborative studies using common standardized methodologies for tumor processing and $R A S$ mutation detection.

In summary, our study supports the use of FOLFIRI plus cetuximab in patients with $R A S$ wild-type tumors and, on the basis of a lack of observed benefit, suggests the exclusion of patients with other RAS mutations. Reserving such first-line treatment for a $R A S$ wild-type population allows the definition of a subgroup more likely to benefit from the addition of cetuximab to FOLFIRI. Molecular testing of tumors for all activating mutations in KRAS and NRAS before considering anti-EGFR therapy is therefore essential in selecting the most effective treatment for patients with mCRC.

\section{AUTHORS' DISCLOSURES OF POTENTIAL CONFLICTS OF INTEREST}

Disclosures provided by the authors are available with this article at www.jco.org.

\section{AUTHOR CONTRIBUTIONS}

Conception and design: Eric Van Cutsem, Heinz-Josef Lenz, Claus-Henning Köhne, Christopher Stroh, Fortunato Ciardiello Provision of study materials or patients: Eric Van Cutsem, Claus-Henning Köhne, Philippe Rougier, Fortunato Ciardiello Collection and assembly of data: Eric Van Cutsem, Claus-Henning Köhne

Data analysis and interpretation: All authors

Manuscript writing: All authors

Final approval of manuscript: All authors

\section{REFERENCES}

1. Van Cutsem $\mathrm{E}$, Köhne $\mathrm{CH}$, Hitre $\mathrm{E}$, et al: Cetuximab and chemotherapy as initial treatment for metastatic colorectal cancer. N Engl J Med 360:1408-1417, 2009

2. Van Cutsem $\mathrm{E}$, Köhne $\mathrm{CH}$, Láng $\mathrm{I}$, et al: Cetuximab plus irinotecan, fluorouracil, and leucovorin as first-line treatment for metastatic colorectal cancer: Updated analysis of overall survival according to tumor KRAS and BRAF mutation status. J Clin Oncol 29:2011-2019, 2011

3. Bokemeyer C, Bondarenko I, Makhson A, et al: Fluorouracil, leucovorin, and oxaliplatin with and without cetuximab in the first-line treatment of metastatic colorectal cancer. J Clin Oncol 27:663671, 2009

4. Bokemeyer C, Bondarenko I, Hartmann JT, et al: Efficacy according to biomarker status of cetuximab plus FOLFOX-4 as first-line treatment for metastatic colorectal cancer: The OPUS study. Ann Oncol 22:1535-1546, 2011

5. Douillard JY, Siena S, Cassidy J, et al: Randomized, phase III trial of panitumumab with infusional fluorouracil, leucovorin, and oxaliplatin (FOLFOX4) versus FOLFOX4 alone as first-line treatment in patients with previously untreated metastatic colorectal cancer: The PRIME study. J Clin Oncol 28:4697-4705, 2010

6. Forbes SA, Bindal N, Bamford S, et al: COSMIC: Mining complete cancer genomes in the Catalogue of Somatic Mutations in Cancer. Nucleic Acids Res 39: D945-D950, 2011

7. Douillard JY, Oliner KS, Siena $S$, et al: Panitumumab-FOLFOX4 treatment and RAS mutations in colorectal cancer. N Engl J Med 369:10231034, 2013

8. Chen CY, Shiesh SC, Wu SJ: Rapid detection of K-ras mutations in bile by peptide nucleic acidmediated PCR clamping and melting curve analysis:
Comparison with restriction fragment length polymorphism analysis. Clin Chem 50:481-489, 2004

9. Dressman D, Yan H, Traverso G, et al: Transforming single DNA molecules into fluorescent magnetic particles for detection and enumeration of genetic variations. Proc Natl Acad Sci U S A 100: 8817-8822, 2003

10. Diehl F, Li M, Dressman D, et al: Detection and quantification of mutations in the plasma of patients with colorectal tumors. Proc Natl Acad Sci U S A 102:16368-16373, 2005

11. Diehl F, Schmidt K, Durkee KH, et al: Analysis of mutations in DNA isolated from plasma and stool of colorectal cancer patients. Gastroenterology 135 : 489-498, 2008

12. Kaplan EL, Meier P: Nonparametric estimation from incomplete observations. J Am Stat Assoc 53:457-481, 1958

13. Bokemeyer $\mathrm{C}$, Köhne $\mathrm{CH}$, Ciardiello $\mathrm{F}$, et al: Treatment outcome according to tumor RAS mutation status in OPUS study patients with metastatic colorectal cancer (mCRC) randomized to FOLFOX4 with/without cetuximab. J Clin Oncol 32, 2014 (suppl 15s; abstr 3505)

14. Peeters M, Price TJ, Cervantes $A$, et al: Final results from a randomized phase 3 study of FOLFIRI \pm panitumumab for second-line treatment of metastatic colorectal cancer. Ann Oncol 25:107-116, 2014

15. Peeters M, Oliner KS, Price TJ, et al: Analysis of KRAS/NRAS mutations in phase 3 study 20050181 of panitumumab (pmab) plus FOLFIRI versus FOLFIRI for second-line treatment (tx) of metastatic colorectal cancer (mCRC). J Clin Oncol 32, 2014 (suppl; abstr LBA387)

16. National Comprehensive Cancer Network: NCCN Guidelines Colon Cancer (version 2.2015). http://www.nccn.org/professionals/physician_gls/ $\mathrm{f}$ guidelines.asp
17. Misale S, Yaeger R, Hobor S, et al: Emergence of KRAS mutations and acquired resistance to anti-EGFR therapy in colorectal cancer. Nature 486:532-536, 2012

18. Schwartzberg LS, Rivera F, Karthaus M, et al: PEAK: A andomized, multicenter phase II study of panitumumab plus modified fluorouracil, leucovorin, and oxaliplatin (mFOLFOX6) or bevacizumab plus mFOLFOX6 in patients with previously untreated, unresectable, wild-type KRAS exon 2 metastatic colorectal cancer. J Clin Oncol 32: 2240-2247, 2014

19. Stintzing $S$, Jung $A$, Rossius $L$, et al: Analysis of KRAS/NRAS and BRAF mutations in FIRE-3: A randomized phase III study of FOLFIRI plus cetuximab or bevacizumab as first-line treatment for wildtype (WT) KRAS (exon 2) metastatic colorectal cancer (mCRC) patients. Presented at the European Cancer Congress, Amsterdam, the Netherlands, September 27-October 1, 2013 (abstr LBA17)

20. Parsons BL, Myers MB: Personalized cancer treatment and the myth of KRAS wild-type colon tumors. Discov Med 15:259-267, 2013

21. Pekin D, Normand C, Kotsopoulos S, et al: Detection and quantification of minority subclones of KRAS on metastatic colorectal cancers by digital microfluidics: Therapeutic implications. Cancer Res 73, 2013 (abstr 4211)

22. Tougeron D, Lecomte $T$, Pagès JC, et al: Effect of low-frequency KRAS mutations on the response to anti-EGFR therapy in metastatic colorectal cancer. Ann Oncol 24:1267-1273, 2013

23. Taly $V$, Laurent-Puig $P$, Pekin $D$, et al: Clinical significance of low frequency KRAS and BRAF subclones for advanced colon cancer management. Presented at the American Association for Cancer Research Annual Meeting, San Diego, CA, April 5-9, 2014 


\section{AUTHORS' DISCLOSURES OF POTENTIAL CONFLICTS OF INTEREST}

Fluorouracil, Leucovorin, and Irinotecan Plus Cetuximab Treatment and RAS Mutations in Colorectal Cancer

The following represents disclosure information provided by authors of this manuscript. All relationships are considered compensated. Relationships are self-held unless noted. I = Immediate Family Member, Inst = My Institution. Relationships may not relate to the subject matter of this manuscript. For more information about ASCO's conflict of interest policy, please refer to www.asco.org/rwc or jco.ascopubs.org/site/ifc.

\section{Eric Van Cutsem}

Research Funding: Amgen (Inst), Bayer (Inst), Boehringer Ingelheim (Inst), Eli Lilly (Inst), Merck Serono (Inst), Novartis (Inst), Roche (Inst), sanofi-aventis (Inst)

\section{Heinz-Josef Lenz}

Honoraria: Merck KGaA, Bristol-Myers Squibb

Consulting or Advisory Role: Merck KGaA, Bristol-Myers Squibb

Research Funding: Merck KGaA, Bristol-Myers Squibb

Travel, Accommodations, Expenses: Merck KGaA, Bristol-Myers Squibb

Claus-Henning Köhne

Honoraria: Merck KGaA, Amgen, Bristol-Myers Squibb, Roche, Pfizer, Bayer

Research Funding: Merck KGaA, Bayer, Novartis, Roche

\section{Volker Heinemann}

Honoraria: Merck KGaA, Roche, Amgen, sanofi-aventis

Consulting or Advisory Role: Merck KGaA, Amgen, Roche, sanofi-aventis

Speakers' Bureau: Merck KGaA, Amgen, Roche, sanofi-aventis

Research Funding: Merck KGaA, Roche, Amgen, sanofi-aventis Expert Testimony: Merck KGaA, Roche, Amgen, sanofi-aventis Travel, Accommodations, Expenses: Merck KGaA, Roche, Amgen, sanofi-aventis

\section{Sabine Tejpar}

Honoraria: Merck Serono, Sanofi Regeneron

Consulting or Advisory Role: Merck Serono, Sanofi Regeneron
Research Funding: Merck serono (Inst), Sanofi Regeneron (Inst) Travel, Accommodations, Expenses: Merck Serono

Ivan Melezínek

Employment: Merck KGaA

Frank Beier

Employment: Merck KGaA

Travel, Accommodations, Expenses: Merck KGaA

Christopher Stroh

Employment: Merck KGaA

Philippe Rougier

Honoraria: Eli Lilly, Novartis, sanofi-aventis, Ipsen

Consulting or Advisory Role: sanofi-aventis, Lilly

Research Funding: Kephren/Keocyt, Eli Lilly, Ipsen, Novartis, sanofi-aventis, Bayer

Travel, Accommodations, Expenses: sanofi-aventis

J. Han van Krieken

Honoraria: Merck KGaA, Amgen, GlaxoSmithKline

Consulting or Advisory Role: Merck KGaA, Amgen, GlaxoSmithKline

Research Funding: Merck KGaA, Amgen

Travel, Accommodations, Expenses: Merck KGaA, Amgen,

GlaxoSmithKline

Fortunato Ciardiello

Honoraria: Merck KGaA, Roche, Bayer

Consulting or Advisory Role: Merck KGaA, Roche, Bayer, Astellas

Research Funding: Merck KGaA, AstraZeneca, Bayer 


\section{Acknowledgment}

We thank Jim Heighway, Cancer Communications and Consultancy, Knutsford, United Kingdom, for providing medical writing services on behalf of the study sponsor. These included drafting and amending an initial outline under the guidance of the authors and subsequently expanding this outline to a full manuscript draft, which he later amended according to further direction from the authors. 